\title{
Positive effects of ocrelizumab in patients with familial mediterranean fever and coexistent multiple sclerosis
}

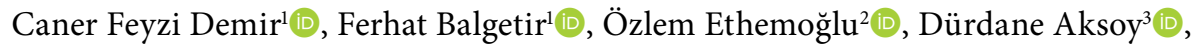 \\ Filiz Aktaş ${ }^{4}$, Süleyman Serdar Koca ${ }^{5}$, Mehmet Fatih Yetkin ${ }^{6}$, İrem Taşc1 ${ }^{7}$ \\ ${ }^{1}$ Department of Neurology, Firat University, Faculty of Medicine, Elazığ, Turkey \\ ${ }^{2}$ Department of Neurology, Harran University, Faculty of Medicine, Şanliurfa, Turkey \\ ${ }^{3}$ Department of Neurology, Gaziosmanpaşa University, Faculty of Medicine, Tokat, Turkey \\ ${ }^{4}$ Department of Neurology, Konya Training and Research Hospital, Konya, Turkey \\ ${ }^{5}$ Department of Internal Medicine, Division of Rheumatology, Firat University, Faculty of Medicine, Elazı̆̆, Turkey \\ ${ }^{6}$ Department of Neurology, Erciyes University, Faculty of Medicine, Kayseri, Turkey \\ ${ }^{7}$ Department of Neurology, Malatya Training and Research Hospital, Malatya, Turkey
}

\begin{abstract}
Objectives: This study aims to evaluate the effects of ocrelizumab (OCZ) on familial Mediterranean fever (FMF) attacks in multiple sclerosis (MS) patients with FMF (MS+FMF patients).

Patients and methods: This retrospective observational study included 11 patients ( 2 males, 9 females; mean age $46.6 \pm 9.2$; range, 22 to 55 years) with MS+FMF hospitalized between January 2016 and July 2019. Demographic, clinical, and laboratory parameters and patient reported outcomes were analyzed in patients treated with OCZ for 18 months.

Results: Combining OCZ with colchicine in MS+FMF patients significantly reduced the frequency of FMF attacks ( $p=0.003)$ and the frequency of joint attacks $(p=0.002)$. Consistent with the clinical improvement, the maximum serum C-reactive protein levels were significantly decreased after combination therapy compared to before combination therapy $(p=0.003)$. MS+FMF patients reported that FMF disease activity improved after OCZ therapy (Visual Analog Scale [VAS] $74 \pm 9.6$ vs. VAS $46.5 \pm 8.1 \mathrm{~mm}, \mathrm{p}=0.003$ ).

Conclusion: Ocrelizumab therapy led to a prominent decrease in the frequency of FMF attacks, alleviated functional impairment, and improved quality of life in MS+FMF patients.

Keywords: Colchicine-resistant familial Mediterranean fever, familial Mediterranean fever, multiple sclerosis, ocrelizumab.
\end{abstract}

The relationship between multiple sclerosis (MS) and familial Mediterranean fever (FMF) has been reported sporadically. Additionally, the prevalence of FMF in Turkish MS patients is reported to be four times higher than the estimated prevalence in the general population., ${ }^{1,2}$ Mitochondrial energy deficit, demyelination, axonal damage, blood-brain barrier dysfunction and recurrent inflammation, which all play a role in MS pathogenesis, may also be observed in FMF.

Received: May 15, 2020 Accepted: August 11, 2020 Published online: January 14, 2021

Correspondence: Ferhat Balgetir, MD. Fırat Üniversitesi Tıp Fakültesi, Nöroloji Anabilim Dalı, 23119 Elazığ, Türkiye. Tel: +90 533 - 4800994 e-mail: ferhatbalgetir@hotmail.com

\section{Citation:}

Demir CF, Balgetir F, Ethemoğlu Ö, Aksoy D, Aktaş F, Koca SS, et al. Positive effects of ocrelizumab in patients with familial mediterranean fever and coexistent multiple sclerosis. Arch Rheumatol 2021;36(2):219-226.

(02021 Turkish League Against Rheumatism. All rights reserved.

This is an open access article under the terms of the Creative Commons Attribution-NonCommercial License, which permits use, distribution and reproduction in any medium, provided the original work is properly cited and is not used for commercial purposes (http://creativecommons.org/licenses/by-nc/4.0/). 
Familial Mediterranean fever is characterized by an exaggerated and inappropriate inflammatory response that particularly involves the excessive expression of proinflammatory cytokines, such as interleukin 1 (IL-1), tumor necrosis factor alpha (TNF- $\alpha$ ) and IL-6, the activation of nuclear factor kappa $B$ and reduced apoptosis of inflammatory cells. These cytokines also play a role in the pathogenesis of MS. Despite much debate among researchers, cohort studies have indicated a correlational relationship between MS and FMF rather than an incidental relationship. ${ }^{3,4}$

Colchicine reduces the frequency, duration and severity of attacks in patients with FMF. Thus, it is the first-line option and main therapy for the treatment of FMF. However, almost 10\% of FMF patients are resistant or intolerant to colchicine treatment. In such cases, IL-1 inhibitors are second-line therapeutic options. TNF- $\alpha$ inhibitors, IL-6 inhibitors and Janus kinase inhibitors are also potential therapeutic agents for resistance or intolerancy to colchicine in FMF patients. ${ }^{4}$

Ocrelizumab (OCZ) is a humanized anti-cluster of differentiation (CD) 20 monoclonal antibody and has been shown to reduce disease activity in MS patients. Additionally, OCZ is becoming increasingly used in the treatment of patients with relapsing MS, secondary progressive MS and primary progressive MS. ${ }^{5}$ We previously observed that OCZ had therapeutic effects on FMF attacks in MS patients with FMF (MS+FMF patients) and these observations form the basis of this study. Accordingly, in this study, we aimed to evaluate the effects of OCZ on FMF attacks in MS+FMF patients.

\section{PATIENTS AND METHODS}

The current retrospective observational study was conducted at Frrat University Faculty of Medicine to evaluate the clinical and radiological records of hospitalized patients with MS between January 2016 and July 2019 in the central and eastern part of Anatolia, Turkey.

The study included 11 MS+FMF patients (2 males, 9 females; mean age 46.6 \pm 9.2 ; range, 22 to 55 years). The diagnoses of MS and FMF were achieved using the $\mathrm{McDonald}^{6}$ and
Tel-Hashomer diagnostic criteria, ${ }^{7}$ respectively. All patients had completed at least 18 months of OCZ therapy (600 mg dose every 24 weeks) prior to the study. The study protocol was approved by the Firat University Faculty of Medicine Ethics Committee (No: 2019-18/12). A written informed consent was obtained from each patient. The study was conducted in accordance with the principles of the Declaration of Helsinki. The clinical, laboratory, genetic and radiological data of the patients were retrieved from their patient records, and both interviews and clinical examinations were performed regularly.

The inclusion criteria were as follows: a diagnosis of MS based on the 2017 McDonald criteria, a diagnosis of FMF based on the Tel-Hashomer diagnostic criteria, a history of OCZ infusion for a minimum period of 18 months (four doses) and availability of complete medical records containing clinical and radiological data.

The exclusion criteria were as follows: the presence of a neurological disease other than MS, such as neuromyelitis optica or myelin oligodendrocyte glycoprotein antibody-associated demyelination, and history of biological agent therapy other than OCZ.

All patients underwent cerebrospinal fluid examination and met the 2017 revised McDonald criteria. Additionally, clinical and laboratory examinations were performed in each patient to rule out other possible causes of vasculitis in the central nervous system, including polyarteritis nodosa, Behçet's disease, Wegener's granulomatosis, systemic lupus erythematosus, antiphospholipid antibody syndrome, Sjögren's syndrome, giant cell arteritis, Churg-Strauss syndrome and Cogan syndrome.

The treatment period was defined as the interval from the first $\mathrm{OCZ}$ infusion to the last neurological examination. Neurological examinations and laboratory tests were performed once every 12 weeks after the first OCZ infusion. During each examination, neurological disability was assessed using the Expanded Disability Status Scale (EDSS). The EDSS assessment was performed by a neurologist certified in EDSS (https://www.neurostatus.net). Patients receiving OCZ therapy were examined by the internal diseases, infectious diseases and neurology departments, particularly for the 
presence of malignancy, hepatitis and other infectious diseases prior to each infusion (once every 24 weeks). Additionally, a complete brain and spinal (both cervical and thoracic) magnetic resonance imaging (MRI) scan was performed in each patient once every 24 weeks using a Signa $^{\mathrm{TM}} 1.5$ Tesla MRI scanner (General Electric Medical Systems, Milwaukee, WI, USA).

All 11 patients included in the study were concurrently receiving colchicine and $\mathrm{OCZ}$ therapy. They started receiving colchicine therapy at the time when the follow-up for FMF was initiated. Based on the clinical criteria, all patients were found to be heterozygous for at least one mutation in the MEFV genomic region. The severity of FMF was assessed using a disease severity score developed by Mor. ${ }^{8}$ The severity of pain was assessed using a $100 \mathrm{~mm}$ Visual Analog Scale (VAS)..$^{9}$ The response to OCZ therapy was assessed using VAS with a range of 0 to 100 , which indicates the severity of the disease. Laboratory parameters (C-reactive protein [CRP], creatinine and proteinuria) were measured during the study. The efficacy of OCZ was quantified by evaluating the reduction of FMF attack frequency, patient and physician global assessments, and CRP levels; this set of assessments has been used previously in the modified FMF50 scoring system. ${ }^{10}$

\section{Statistical analysis}

Statistical analysis was performed using the IBM SPSS version 22.0 software (IBM Corp., Armonk, NY, USA). Values were calculated as means and ranges (min-max). Study parameters were not normally distributed, so the Wilcoxon signed rank test was used to compare data before OCZ and after OCZ treatment, with $p$ values of $<0.05$ considered statistically significant.

\section{RESULTS}

The demographic and clinical characteristics of the patients were presented in Table 1 . Clinical characteristics of attacks were presented in Table 2. Two patients (\#1 and \#2) were siblings. The mean age at the time of MS diagnosis was $25.6 \pm 5.8$ (range, 16 to 35 ) years, while the mean age at the time of diagnosis of FMF was 15.7 \pm 11.9 (range, 9 to 48 ) years. All 11 patients had positive family histories of FMF and four patients had positive family histories of MS.

All patients had a diagnosis of secondary progressive MS (SPMS), all experiencing cerebral and spinal involvement. Of these, 10 patients had type II oligoclonal banding $(\mathrm{OCB})$ and one patient had type III OCB. All patients had immunoglobulin $\mathrm{G}$ indices higher than 0.7.

The mean EDSS score was $5.5 \pm 0.6$ (range, 4.5 to 6.5 ) and it did not differ significantly at any point during the 18-month period. All patients had received prophylaxis for MS, including glatiramer acetate, interferon (IFN) beta, azathioprine, dimethyl fumarate, fingolimod and methotrexate. All patients had a suboptimal response to prior treatment for MS and continued taking a stable dose of colchicine for FMF throughout the treatment and follow-up periods.

Prior to OCZ treatment, FMF attacks were severe in four, moderate in five, and mild in two patients, respectively. All patients were receiving colchicine $(1.5 \mathrm{mg} /$ day $)$ for FMF treatment throughout the study. Five patients showed a good response to the colchicine therapy before OCZ treatment, with the remaining six showing a partial response (Table 1).

Following the OCZ therapy, the severity of FMF attacks decreased significantly in six patients, whereas the duration and frequency of FMF attacks decreased in all patients (Tables 1 and 3). However, the severity of attacks remained moderate in two patients and the symptoms were mild in two patients who had no further attacks.

Although the patients were receiving colchicine at a tolerable dose and had regular follow-up visits, each patient had at least one FMF attack per month before OCZ treatment (Table 3). In this study, patients who received OCZ therapy for MS and colchicine therapy for the accompanying FMF experienced a significant reduction in the frequency of FMF attacks $(p=0.003)$ and the frequency of joint attacks $(p=0.002)$ after combination treatment compared to before combination treatment (Table 3). Consistent with the clinical improvement, the maximum serum CRP levels were significantly decreased after combination therapy $(6.13 \pm 1.54 \mathrm{mg} / \mathrm{L})$ compared to before combination therapy $(8.71 \pm 1.94 \mathrm{mg} / \mathrm{L})$ 


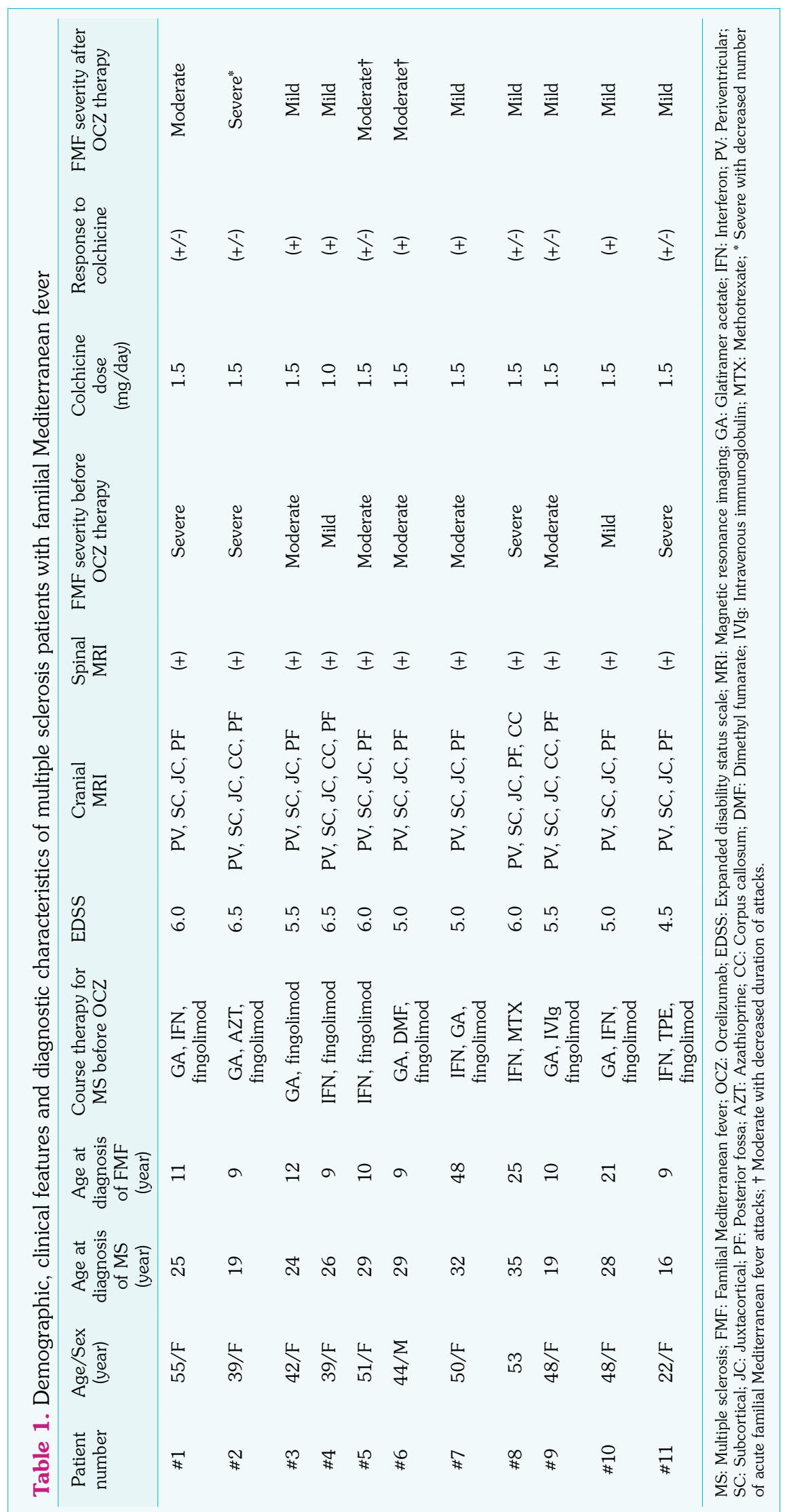


Table 2. Clinical features of familial Mediterranean fever attacks $(n=11)$

\begin{tabular}{lcc}
\hline & $\mathrm{n}$ & $\%$ \\
\hline Fever & 11 & 100 \\
Abdominal pain & 11 & 100 \\
Pleuritis & 6 & 54.55 \\
Pericarditis & 2 & 18.18 \\
Arthritis & 6 & 54.55 \\
Erysipelas-like skin lesion & 3 & 27.27 \\
Amyloidosis & 5 & 45.45
\end{tabular}

(Table 3, $p=0.003$ ). MS+FMF patients reported that FMF disease activity improved after OCZ therapy (VAS $74 \pm 9.6$ vs. VAS $46.5 \pm 8.1 \mathrm{~mm}, \mathrm{p}=0.003$ ) (Table 3). Additionally, physician-reported FMF disease activity significantly improved (VAS 75 \pm 10 vs. VAS $49.1 \pm 8.6 \mathrm{~mm}, \mathrm{p}=0.003$ ) after 18 months of OCZ therapy (Table 3). All patients showed significant improvement in the modified FMF50 score (Table 3).

\section{DISCUSSION}

Some studies showed that the rate of FMF in MS patients was two or four times higher than the expected prevalence in Turkey. ${ }^{1,11,12} \mathrm{~A}$ previous study systematically screened a cohort of 157 MS patients for FMF-specific mutations and detected mutations of the MEFV gene in 19 patients, among whom 15 had at least one symptom suggestive of FMF. ${ }^{13}$ It was found that the frequency of the FMF-MS complex is $0.075 \%$ in a pool of 12,000 FMF patients in Israel. ${ }^{14}$ It seems that FMF patients are predisposed to develop MS, consistent with previous findings of increased co-occurrence of FMF with other auto-inflammatory diseases. Some of these previous studies have suggested that genetic mutations related to FMF may have negative effect on the course of MS. ${ }^{14-16}$ Larger and longer follow-up studies would be needed to assess the effect of this relationship.

The primary aim of the present study was to investigate the efficacy and safety of OCZ therapy on MS+FMF in patients with SPMS, and also to explore whether the treatment has any effect in the reduction of acute FMF attacks.
Our patients did not differ from the general progressive MS population in terms of clinical findings, progression and response to $\mathrm{OCZ}$ therapy. Treatment of MS was associated with a good response, and no clinical activity occurred in any patients. In the case of stable disease, an annual MRI is recommended routinely to monitor disease activity and treatment response. ${ }^{17}$ In this study, further MRIs were obtained every six months during 18 months to assess the activity of progressive disease despite OCZ treatment. No MS patients experienced clinical and/or MRI disease activity during the $\mathrm{OCZ}$ therapy (during 18 months of the study). All patients satisfied combined clinical and radiologic criteria of no evidence of disease activity except patient 3. In patient 3, a new T2 lesion was observed with routine MRI scans, without any new neurologic symptoms.

The efficacy of prophylactic colchicine therapy in controlling febrile attacks in FMF patients and in preventing the most devastating complication of FMF (i.e., amyloidosis) has been known for the last five decades. ${ }^{18}$ In addition, the identification of the causative gene on the short arm of chromosome 16 (MEFV gene) allowed a better understanding of the pathogenesis of FMF. ${ }^{19}$ Following these developments, the recommended treatment options for FMF and other related diseases were revised. Accumulating evidence suggests that MEFV mutations could be associated with increased IL-1 $\beta$ activation. ${ }^{20}$ After the identification of the role of pyrin and IL-1 $\beta$ in the pathogenesis of FMF, IL- 1 has become a promising target for controlling inflammation in patients with inadequate response to colchicine.

On the other hand, biological agents that antagonize IL-1, IL-6, and TNF- $\alpha$ are used in patients unresponsive to colchicine due to their inhibitive effects. ${ }^{21}$ Additionally, numerous alternatives have been recently tried for the reduction of inflammation in FMF, including IFN alpha (IFN- $\alpha$ ), anakinra, rilonacept, etanercept, infliximab, canakinumab, tocilizumab, thalidomide, nonsteroidal anti-inflammatory drugs, and dietary and herbal supplements. ${ }^{21}$

To our knowledge, there are some controlled trials published in the literature reporting on the use of biological agents in acute attacks of FMF, of which one study investigated the 


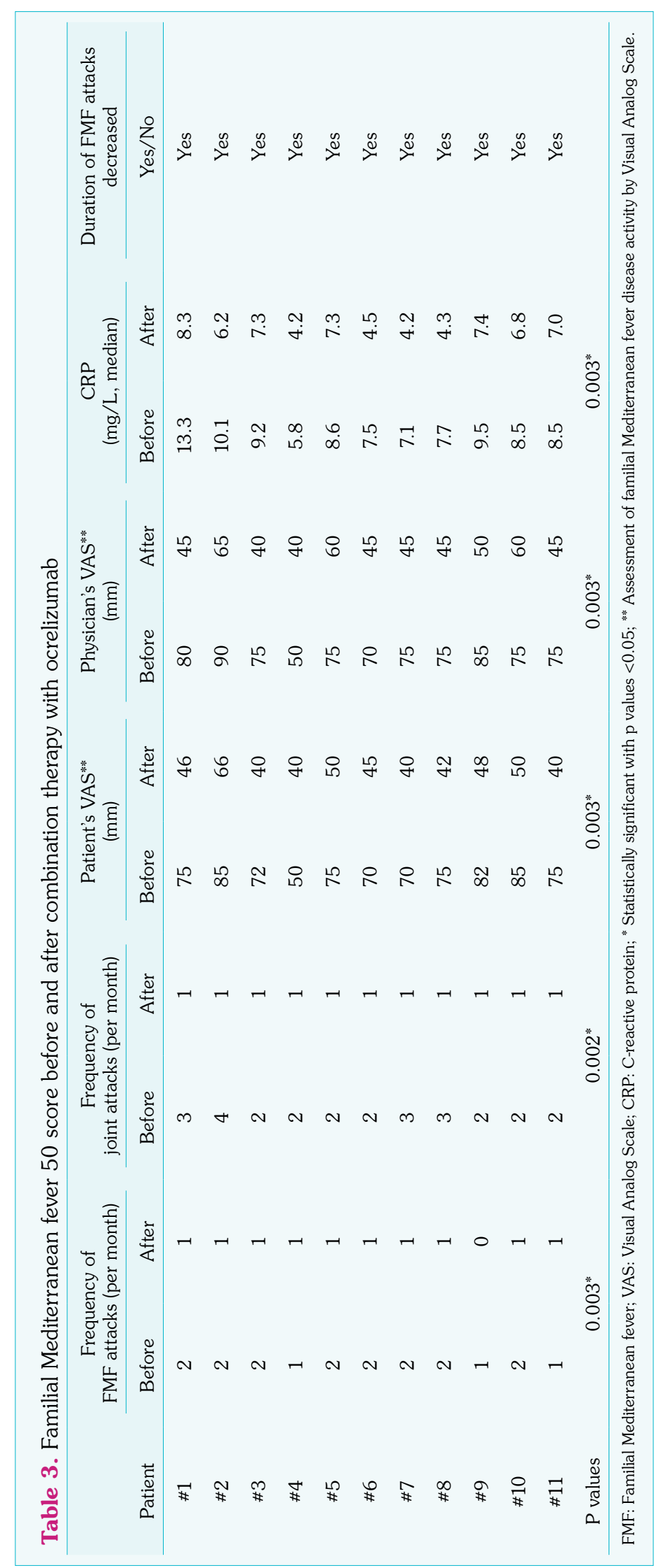


efficacy of IFN- $\alpha^{22}$ and the other investigated the effectiveness of IL- 1 trap (rilonacept). ${ }^{23}$ Additionally, there is another trial exploring the efficacy of 16 - and 40-week courses of canakinumab, which is a human anti-IL-1 $\beta$ monoclonal antibody. ${ }^{24}$ Moreover, there is a double-blind placebo-controlled trial which investigated the efficacy and safety of anakinra, a recombinant form of the human IL-1 receptor antagonist, for the treatment of colchicine-resistant FMF. ${ }^{25,26}$

Ocrelizumab has been shown to selectively deplete CD20-expressing B cells. ${ }^{27}$ Although CD20 is known to be expressed by B cells, it is also expressed by a small subset of $\mathrm{T}$ cells $\left(\mathrm{CD}^{+}, \mathrm{CD}^{2} 0^{+} \mathrm{T}\right.$ cells). These $\mathrm{CD} 20^{+} \mathrm{T}$ cells display a distinct phenotype compared to most CD20 $\mathrm{T}$ cells, with an elevated percentage of $\mathrm{CD}^{+}$and $\mathrm{CD}^{+} 5 \mathrm{RO}^{+}$cells. ${ }^{28} \mathrm{CD}^{2} 0^{+} \mathrm{T}$ cells comprise a highly activated cell population that is characterized by the enhanced production of proinflammatory cytokines (e.g., TNF- $\alpha$, IL-1 $\beta$ and IL-17) upon activation. ${ }^{29}$ Meaningfully, it appears that OCZ not only depletes B cells, but also depletes proinflammatory cytokines that are produced by $\mathrm{CD} 20^{+} \mathrm{T}$ cells, including IL-1.

Conducting randomized studies and obtaining conclusive results from MS+FMF patients are highly challenging due to the rareness of such patients. The small sample size is hence a limitation of our study. Other limitations include the observational design, as well as the heterogeneity of MS+FMF patients. Further studies with larger sample sizes and longitudinal designs are needed to elucidate the role of mutations and polymorphisms, and the therapeutic effect of OCZ on FMF patients without MS.

Fatigue, poor sense of well being and bodily pain may not be only due to FMF, but also MS. Therefore, we did not use the health-related quality of life (HRQOL) parameter to its fullest extent while assessing the physical and mental health of our patients. Instead, we only used the visual analog rating scale component of HRQOL. This issue may be considered another limitation of this study.

In conclusion, the results indicate that $\mathrm{OCZ}$ therapy decreases the frequency of FMF attacks, improves functional impairment and increases the quality of life in MS+FMF patients. Further studies involving extensive observation are required to determine the long-term safety and efficacy of OCZ in patients with FMF.

\section{Declaration of conflicting interests}

The authors declared no conflicts of interest with respect to the authorship and/or publication of this article.

\section{Funding}

The authors received no financial support for the research and/or authorship of this article.

\section{REFERENCES}

1. Akman-Demir G, Gul A, Gurol E, Ozdogan $\mathrm{H}$, Bahar $\mathrm{S}$, Oge $\mathrm{AE}$, et al. Inflammatory/ demyelinating central nervous system involvement in familial Mediterranean fever (FMF): coincidence or association? J Neurol 2006;253:928-34.

2. Unal A, Dursun A, Emre U, Tascilar NF, Ankarali $\mathrm{H}$. Evaluation of common mutations in the Mediterranean fever gene in Multiple Sclerosis patients: is it a susceptibility gene? J Neurol Sci 2010;294:38-42.

3. Alpayci M, Bozan N, Erdem S, Gunes M, Erden $\mathrm{M}$. The possible underlying pathophysiological mechanisms for development of multiple sclerosis in familial Mediterranean fever. Med Hypotheses 2012;78:717-20.

4. El Hasbani G, Jawad A, Uthman I. Update on the management of colchicine resistant Familial Mediterranean Fever (FMF). Orphanet J Rare Dis 2019;14:224.

5. Turner B, Cree BAC, Kappos L, Montalban X, Papeix C, Wolinsky JS, et al. Ocrelizumab efficacy in subgroups of patients with relapsing multiple sclerosis. J Neurol 2019;266:1182-93.

6. Thompson AJ, Banwell BL, Barkhof F, Carroll WM, Coetzee T, Comi G, et al. Diagnosis of multiple sclerosis: 2017 revisions of the McDonald criteria. Lancet Neurol 2018;17:162-73.

7. Pras M. Familial Mediterranean fever: from the clinical syndrome to the cloning of the pyrin gene. Scand $\mathrm{J}$ Rheumatol 1998;27:92-7.

8. Mor A, Shinar Y, Zaks N, Langevitz P, Chetrit A, Shtrasburg $S$, et al. Evaluation of disease severity in familial Mediterranean fever. Semin Arthritis Rheum 2005;35:57-64.

9. Price DD, McGrath PA, Rafii A, Buckingham B. The validation of visual analogue scales as ratio scale measures for chronic and experimental pain. Pain 1983;17:45-56. 
10. Ozen S, Demirkaya E, Duzova A, Erdogan O, Erken E, Gul A, et al. FMF50: a score for assessing outcome in familial Mediterranean fever. Ann Rheum Dis 2014;73:897-901.

11. Kalyoncu U, Eker A, Oguz KK, Kurne A, Kalan I, Topcuoglu AM, et al. Familial Mediterranean fever and central nervous system involvement: a case series. Medicine (Baltimore) 2010;89:75-84.

12. Balcı-Peynircioğlu B, Kaya-Akça Ü, Arıcı ZS, Avcı E, Akkaya-Ulum ZY, Karadağ Ö, et al. Comorbidities in familial Mediterranean fever: analysis of 2000 genetically confirmed patients. Rheumatology (Oxford) 2020;59:1372-80.

13. Kümpfel T, Gerdes LA, Wacker T, Blaschek A, Havla J, Krumbholz M, et al. Familial Mediterranean fever-associated mutation pyrin E148Q as a potential risk factor for multiple sclerosis. Mult Scler 2012;18:1229-38.

14. Yahalom G, Kivity S, Lidar M, Vaknin-Dembinsky A, Karussis D, Flechter S, et al. Familial Mediterranean fever (FMF) and multiple sclerosis: an association study in one of the world's largest FMF cohorts. Eur $\mathrm{J}$ Neurol 2011;18:1146-50.

15. Ozaras N, Akman-Demir G, Ustek D, Eraksoy M, Abaci N, Gul A. Does FMF gene exist in multiple sclerosis and contribute to the progression? Neurology Asia 2014;19:235-6.

16. Shinar Y, Livneh A, Villa Y, Pinhasov A, Zeitoun I, Kogan A, et al. Common mutations in the familial Mediterranean fever gene associate with rapid progression to disability in non-Ashkenazi Jewish multiple sclerosis patients. Genes Immun 2003;4:197-203.

17. Kaunzner UW, Gauthier SA. MRI in the assessment and monitoring of multiple sclerosis: an update on best practice. Ther Adv Neurol Disord 2017;10:247-61.

18. Dinarello CA, Wolff SM, Goldfinger SE, Dale DC, Alling DW. Colchicine therapy for familial mediterranean fever. A double-blind trial. N Engl J Med 1974;291:934-7.
19. French FMF Consortium. A candidate gene for familial Mediterranean fever. Nat Genet 1997;17:25-31.

20. Saito M, Nishikomori R, Kambe N. [Familial Mediterranean fever: MEFV gene mutations and treatment]. Nihon Rinsho Meneki Gakkai Kaishi 2007;30:78-85.

21. Alghamdi M. Familial Mediterranean fever, review of the literature. Clin Rheumatol 2017;36:1707-13.

22. Wu B, Xu T, Li Y, Yin X. Interventions for reducing inflammation in familial Mediterranean fever. Cochrane Database Syst Rev 2015;3:CD010893.

23. Tunca M, Akar S, Soytürk M, Kirkali G, Resmi $\mathrm{H}$, Akhunlar $\mathrm{H}$, et al. The effect of interferon alpha administration on acute attacks of familial Mediterranean fever: A double-blind, placebocontrolled trial. Clin Exp Rheumatol 2004;22(4 Suppl 34):S37-40.

24. Ozdogan H, Ugurlu S. Canakinumab for the treatment of familial Mediterranean fever. Expert Rev Clin Immunol 2017;13:393-404.

25. Hashkes PJ, Spalding SJ, Giannini EH, Huang B, Johnson A, Park G, et al. Rilonacept for colchicineresistant or -intolerant familial Mediterranean fever: a randomized trial. Ann Intern Med 2012;157:533-41.

26. Ben-Zvi I, Kukuy O, Giat E, Pras E, Feld O, Kivity $S$, et al. Anakinra for colchicine-resistant familial mediterranean fever: A randomized, double-blind, placebo-controlled trial. Arthritis Rheumatol 2017;69:854-62.

27. Montalban X, Hauser SL, Kappos L, Arnold DL, BarOr A, Comi G, et al. Ocrelizumab versus Placebo in Primary Progressive Multiple Sclerosis. N Engl J Med 2017;376:209-20.

28. Hultin LE, Hausner MA, Hultin PM, Giorgi JV. CD20 (pan-B cell) antigen is expressed at a low level on a subpopulation of human $\mathrm{T}$ lymphocytes. Cytometry 1993;14:196-204.

29. Schuh E, Berer K, Mulazzani M, Feil K, Meinl I, Lahm $\mathrm{H}$, et al. Features of Human CD3+CD20+ T Cells. J Immunol 2016;197:1111-7. 